\title{
Multivariate dependent interval finite element analysis via convex hull pair constructions and the Extended Transformation Method
}

\author{
Matthias Faes ${ }^{\mathrm{a}, *}$, David Moens ${ }^{\mathrm{a}}$ \\ ${ }^{a} K U$ Leuven, Department of Mechanical Engineering, Technology campus De Nayer, Jan \\ De Nayerlaan 5, St.-Katelijne-Waver, Belgium
}

\begin{abstract}
Classical (independent) interval analysis considers a hyper-cubic input space consisting of independent intervals. This stems from the inability of intervals to model dependence and results in a serious over-conservatism when no physical guarantee of independence of these parameters exists. In a spatial context, dependence of one model parameter over the model domain is usually modelled using a series expansion over a set of basis functions that interpolate a set of globally defined intervals to local (coupled) uncertainty. However, the application of basis functions is not always appropriate to model dependence, especially when such dependence does not have a spatial nature but is rather scalar. This paper therefore presents a flexible approach for the modelling of dependent intervals that is also applicable to multivariate problems. Specifically, it is proposed to construct the dependence structure in a similar approach to copula pair constructions, yielding a limited set of 2-dimensional dependence functions. Furthermore, the well-known Transformation Method is extended to the case of dependent interval analysis. The applied case studies indicate the flexibility and performance of the method.

Keywords: Interval analysis, Dependent intervals, Copula pair Constructions, non-probabilistic analysis, Transformation method, imprecise probability
\end{abstract}

\footnotetext{
${ }^{*}$ Corresponding author

Email address: matthias.faes@kuleuven.be (Matthias Faes)
}

Preprint submitted to Computer Methods in Applied Mechanics and EngineeringDecember 13, 2018 


\section{Introduction} techniques (see e.g., [1] for a recent treatment).

Underlying these interval computations, a system of sets of partial differential equations (PDE) needs to be solved repeatedly. The approximative solution of these PDE's is usually provided by means of a numerical model $\mathcal{M}(\boldsymbol{x})$, parametrized by a parameter vector $\boldsymbol{x}(\mathbf{r}) \in \mathcal{X} \subset \mathbb{R}^{d_{i}}$ with $\mathcal{X}$ the set of physically admissible parameters and $d_{i} \in \mathbb{N}$. For example, $\boldsymbol{x}(\mathbf{r})$ may contain inertial moments, clamping stiffness values or constitutive material parameters as a function of a spatial coordinate $\mathbf{r} \in \Omega \subset \mathbb{R}^{d_{\Omega}}$ over the model domain $\Omega$ with dimension $d_{\Omega} \in \mathbb{N}, d_{\Omega} \leq 4$. In case $\mathcal{M}(\boldsymbol{x})$ is constructed following a finite element approach, $\Omega$ is discretised by means of a set of finite elements, yielding $d_{d}$ structural degrees of freedom (DOF). The model $\mathcal{M}(\boldsymbol{x})$ provides a vector of model responses $\boldsymbol{y}(\mathbf{r}) \in \mathcal{Y} \subset \mathbb{R}^{d_{o}}$, with $\mathcal{Y}$ the set of admissible model responses and $d_{o} \in \mathbb{N}$, through a set of function operators $m_{i}, i=1, \ldots, d_{o}$, which are defined as:

$$
\mathcal{M}(\boldsymbol{x}): y_{i}(\mathbf{r})=m_{i}(\boldsymbol{x}(\mathbf{r})) \quad i=1, \ldots, d_{o}
$$

with $m_{i}: \mathbb{R}^{d_{i}} \mapsto \mathbb{R}$ Note that the dependence of $\boldsymbol{y}$ on $\mathbf{r}$ is only valid when nodal or elemental responses are considered. This is for example not the case when $\boldsymbol{y}$ consists of eigenfrequencies.

One of the key challenges in the application of interval theoretical approaches for modelling uncertainty in finite element models, is the inability of intervals to account for dependence between multiple uncertain parameters. A large body of literature is therefore dedicated to minimize the over-estimation of interval 
models due to this independence, following an element-by-element approach [3] or following affine arithmetic 4]. However, in some cases also dependence between multiple $x_{i}(\boldsymbol{r}), i=1, \ldots, d_{i}$ (inter-uncertainty) or $\boldsymbol{x}\left(r_{i}\right), i=1, \ldots, d_{\Omega}$ (intra-uncertainty) has to be considered to allow for the realistic modelling of the non-deterministic structure of the model parameters. In the context of spatial dependence of a single parameter, recent work of the authors focussed on modelling spatial dependence via interval fields [5]. Following the method presented in [5], an interval field $\boldsymbol{x}^{\boldsymbol{I}}(\mathbf{r}): \Omega \times \mathbb{I}^{n_{b}} \mapsto \mathbb{I} \mathbb{R}$ is modelled as a series expansion, where local uncertainty is modelled using $n_{b} \in \mathbb{N}$ globally defined independent interval scalars $\boldsymbol{\alpha}^{I} \in \mathbb{I R}^{n_{b}}$ and basis functions $\boldsymbol{\psi}(\mathbf{r}): \Omega \mapsto \mathbb{R}$ :

$$
\boldsymbol{x}^{\boldsymbol{I}}(\mathbf{r})=\sum_{i=1}^{n_{b}} \boldsymbol{\psi}(\mathbf{r}) \cdot \alpha_{i}^{I}
$$

with $\mathbb{I R}^{n_{b}}$ the domain of real-valued interval vectors of dimension $n_{b}$. This framework for the modelling of spatial uncertainty modelling was recently applied in the context of inverse uncertainty quantification [6, 7] and the modelling of various dynamic phenomena [8, 9, 10, as well as additively manufactured plastic components [1]. Also alternative formulations for modelling spatial uncertainty in an interval context have been proposed by other authors [12, 13.

However, while being valuable in the context of modelling spatial phenomena, when considering the dependence between multiple physical parameters of a numerical model, such a weighting approach might not be appropriate. For instance, considering parameters such as material strength and stiffness of a component that is produced using a casting approach, typically a positive dependence between such parameters would be expected. Conversely, when looking at the width and thickness of such a part, a negative dependence could be introduced due to gravitational effects. This very simple example illustrates the often highly complex nature of the combination of different dependence structures throughout a numerical model, especially when a higher-level dependence between strength/stiffness and width/thickness exists. Hence, a simple weighting of interval scalars using a single set of basis functions might in that case prove to be too inflexible to allow for the accurate and realistic modelling 
of this dependence.

An alternative approach in this context is based on the the set-theoretical work of Elishakoff and co-workers, who throughout recent years introduced several set-theoretical approaches to cope with dependence in a non-probabilistic way [14, 15, 16, 17]. Following the most basic approach, the dependence can be represented using a $d$-dimensional hyper-ellipsoid which should abide by some minimum volume property. Also extensions towards Lamé curves and other, nodal, convex sets were introduced in recent years [18, 19. However, while providing the analyst with an intuitive tool, the underlying assumption is still that all parameters are governed by a single underlying dependence structure. A solution hereto could be to only consider pair-wise dependence, but this neglects possible higher-order dependence structures between multiple model quantities.

In the context of probabilistic modelling of uncertainty, techniques based on Copula 20] are being applied widely, for instance in the modelling of dependence in system reliability 21, naval engineering 22] or inverse Bayesian random field quantification 23. Application of these methods extend also far beyond the engineering realm with for example wide application in financial mathematics [24] and machine learning [25]. These methods indeed provide a flexible tool to model complex dependencies in an intuitive and elegant way, but sampling from a copula in $d>2$ proves to be a daunting task [21]. Furthermore, also here the argumentation holds that it is questionable to model all dependency using a single Copula family (i.e., dependence structure). As a possible solution hereto, Copula pair constructions were introduced in the seminal papers of Bedford and Cooke [26, 27] and further elaborated on by Aas [28. The core idea hereof is to decompose the multivariate, higher-order dependencies as a product of marginal distributions, a set of 2-dimensional unconditional Copula and a set of conditional Copula, allowing for the definition of a dependence structure for each combination of parameters. However, these methods rely heavily on the underlying statistical derivations and hence, it is unclear how these methods should be applied in an interval context without violating the interval paradigm where only crisp bounds on the uncertain quantities are considered. 
This paper therefore explores the application of copula pair construction approaches for the modelling of dependence between uncertain parameters of a FE model that are modelled as intervals. Specifically, it is aimed at introducing a generic set-theoretical method that allows an analyst to define a high-order dependence structure as a product of 2-dimensional, possibly non-convex, admissible sets that bound the combination of parameter values within $\boldsymbol{x}^{\boldsymbol{I}}$. Hereto, the bounded global optimisation problem that underlies typical interval computations is recast into a non-linear constrained global optimisation problem to accommodate these higher-order dependence structures. The paper is structured as follows. In section 2 , a concise introduction to copula in a probabilistic context is presented. Then, section 3 proposes a new set-theoretical method to propagate multivariate interval uncertainty with dependence between the interval valued parameters. Section 5 and 6 present two case studies to illustrate the application of these ideas to both an academic case study as well as a realistic finite element model. Conclusions are listed in section 7.

\section{Copula in a probabilistic context}

This section introduces Copula and Copula pair constructions in a concise way. It is not intended to provide the reader with a mathematically thorough introduction to Copula since this lies outside the scope of this paper, but rather to convey the general ideas that are needed in the development of the new interval method.

\subsection{Copula}

A Copula $C$ is a function that constructs a joint cumulative distribution function $F_{1: d_{i}}\left(x_{1}, x_{2}, \ldots x_{d_{i}}\right)$, with $x_{1}, x_{2}, \ldots x_{d_{i}} \in[0,1]^{d_{i}}$ starting from its onedimensional marginal distribution functions $F_{i}, i=1, \ldots, d_{i}$. As such, the modelling of the dependence is decoupled from the modelling of the non-determinism in the model parameters via their marginals. The application of Copula on a bivariate distribution is based on Sklar's theorem [20]:

$$
F_{1: d_{i}}=C_{1: d_{i}}\left(F_{1}\left(x_{1}\right), F_{2}\left(x_{2}\right), \ldots, F_{d_{i}}\left(x_{d_{i}}\right)\right)
$$


$\forall \boldsymbol{x} \in \mathbb{R}^{d_{i}}$, with $C_{1: d_{i}}:[0,1]^{d_{i}} \mapsto[0,1]$ the copula function. In case all $F_{i}, i=$ $1, \ldots, d_{i}$ are continuous, $F_{1: d_{i}}$ is unique. Note that a copula is always contained between the Fréchet-Hoeffding bounds:

$$
\max \left(1-d_{i}+\sum_{i=1}^{d} x_{i}, 0\right) \leq C_{1: d_{i}} \leq \min \left(x_{i}\right)
$$

which bound the dependence between the parameters and correspond to the probability mass lying on the principal diagonals of $[0,1]^{d_{i}}$.

Two types of families are directly applicable to cases where $d_{i}>2$ : Gaussian and Archimedean copula, and hence, attract a lot of scientific and industrial interest. The Gaussian copula is defined as:

$$
F_{G, 1: d_{i}}=\Phi_{d_{i}}\left(\Phi^{-1}\left(u_{1}\right), \ldots, \Phi^{-1}\left(u_{d_{i}}\right)\right)
$$

with $u_{i} \in[0,1]$ a coordinate in standard normal space, $\Phi^{-1}\left(u_{i}\right): \mapsto$ the inverse univariate cumulative distribution function of $u_{i}$ and $\Phi_{d_{i}}: \mapsto$ the cumulative distribution function as $\mathcal{N}(0, R)$ and $R \in \mathbb{R}^{d_{i} \times d_{i}}$ a positive definite correlation matrix. As such, this corresponds to the well-known Nataf function. The definition of Archimedean copula is similar as in eq. (5), with the main difference being that $\Phi^{-1}\left(u_{i}\right)$ and $\Phi_{d_{i}}$ are replaced by a so-called generator function and its inverse [29]. The formulation of the generator function depends on the copula family (e.g., Frank, Gumbel, Plancket and so forth). However, it may not be physically accurate to use the same copula family (i.e., dependence structure) to model the dependence between all combinations of parameters since this dependence is in general not the same. Furthermore, sampling from a copula in $d>2$ proves to be a daunting task [21].

\subsection{Pair Copula Construction}

To overcome the limitations of these regular copula, the $d_{i}$-dimensional density $f\left(x_{1}, \ldots, x_{d_{i}}\right)$ of a random vector $\boldsymbol{X}=\left(X_{1}, X_{2}, \ldots, X_{n}\right)$ is constructed using a product of $d(d-1) / 2$ bivariate (conditional) copula [26, 28]. 
As a first step, expressing eq. (3) for continuous, strictly monotonic marginal density functions $f_{1}, \ldots, f_{n}$ via derivation w.r.t. $x$ yields:

$$
f\left(x_{1}, \ldots, x_{d_{i}}\right)=c_{1, \ldots, d_{i}}\left(f_{1}\left(x_{1}\right), \ldots, f_{n}\left(x_{n}\right)\right) \cdot f_{1}\left(x_{1}\right) \cdots f_{d_{i}}\left(x_{d_{i}}\right)
$$

where $c_{1, \ldots, d_{i}}$ is a uniquely defined $d_{i}$-dimensional copula density function. The copula-pair construction is based on following factorisation of $f\left(x_{1}, \ldots, x_{d_{i}}\right)$ :

$$
\begin{aligned}
f\left(x_{1}, \ldots, x_{d_{i}}\right)= & f_{d_{i}}\left(x_{d_{i}}\right) \cdot f\left(x_{d_{i}-1} \mid x_{d_{i}}\right) \cdot f\left(x_{d_{i}-2} \mid x_{d_{i}-1}, x_{d_{i}}\right) \cdots \\
& f\left(x_{1} \mid x_{2}, \ldots, x_{d_{i}}\right)
\end{aligned}
$$

which is a product of conditional and unconditional marginals. Taking into account eq. (6), each term in eq. (7) can be decomposed as:

$$
f(x \mid \boldsymbol{v})=c_{x v_{j}} \mid \boldsymbol{v}_{-j}\left(F\left(x \mid \boldsymbol{v}_{-j}\right) \mid F\left(v_{j} \mid \boldsymbol{v}_{-j}\right)\right) \cdot f\left(x \mid \boldsymbol{v}_{-j}\right)
$$

for a general vector $\boldsymbol{v} 28$.

Hence, the factorisation given in eq. (7) can be constructed as a product of $d(d-1) / 2$ bivariate (conditional) copula and their marginals [26, 28]. This enables the modelling of dependence between two $x_{i}$ with a much higher degree of flexibility, since different $c_{i j}$ can be employed for all $x_{i}$.

For instance, when considering a three-dimensional random vector $\boldsymbol{X}=$ $\left(X_{1}, X_{2}, X_{3}\right)$ and applying eq. (8), the bivariate densities can be written as:

$$
\begin{array}{rr}
f_{2 \mid 1}\left(x_{2} \mid x_{1}\right) & =c_{12}\left(f_{1}\left(x_{1}\right), f_{2}\left(x_{2}\right)\right) \\
f_{3 \mid 2}\left(x_{3} \mid x_{2}\right) & =c_{32}\left(f_{3}\left(x_{3}\right), f_{2}\left(x_{2}\right)\right) \\
f_{3 \mid 12}\left(x_{3} \mid x_{1}, x_{2}\right) & =c_{13 \mid 2}\left(f_{1 \mid 2}\left(x_{1} \mid x_{2}\right), f_{3 \mid 2}\left(x_{3} \mid x_{2}\right)\right)
\end{array}
$$

which yields:

$$
\begin{aligned}
f\left(x_{1}, x_{2}, x_{3}\right)= & f_{1}\left(x_{1}\right) \cdot f_{2}\left(x_{2}\right) \cdot f_{3}\left(x_{3}\right) \cdot c_{12}\left(f_{1}\left(x_{1}\right), f_{2}\left(x_{2}\right)\right) \cdot \\
& c_{23}\left(f_{2}\left(x_{2}\right), f_{3}\left(x_{3}\right)\right) \cdot c_{13 \mid 2}\left(f_{1 \mid 2}\left(x_{1} \mid x_{2}\right), f_{3 \mid 2}\left(x_{3} \mid x_{2}\right)\right)
\end{aligned}
$$

with $c_{i j \mid k}$ the bivariate copula linking $F_{i}$ and $F_{j}$ conditional on $x_{k}$. For higher $d_{i}$, a similar construction can be made. As can be noted, a large number of constructions can be contrived when $d_{i}$ is large. Most commonly, therefore a set 
of nested trees is used to visualise and enumerate these constructions via graph theory. Such set of nested trees is also referred to as a "vine copula". Following a $\mathfrak{D}$-vine decomposition, the copula can be constructed as:

$$
\begin{aligned}
& f_{1, \ldots, d_{i}}\left(x_{1}, x_{2}, \ldots, x_{d_{i}}\right)=\prod_{k=1}^{d_{i}} f\left(x_{k}\right) \prod_{j=1}^{d_{i}-1} \prod_{i=1}^{d_{i}-j} c_{i, i+j \mid i+1, \ldots, i+j+1} \\
&\left(F\left(x_{i} \mid x_{i+1}, \ldots, x_{i+j-1}\right), F\left(x_{i+j} \mid x_{i+1}, \ldots, x_{i+j-1}\right)\right)
\end{aligned}
$$

Alternatively, the $d_{i}$-dimensional copula can also be represented following a $\mathfrak{C}$-vine decomposition:

$$
\begin{array}{r}
f_{1, \ldots, d_{i}}\left(x_{1}, x_{2}, \ldots, x_{d_{i}}\right)=\prod_{k=1}^{d_{i}} f\left(x_{k}\right) \prod_{j=1}^{d_{i}-1} \prod_{i=1}^{d_{i}-j} \mathcal{D}_{j, j+i \mid 1, \ldots, j-1} \\
\left(F\left(x_{j} \mid x_{j=1}, \ldots, x_{j+1}\right), F\left(x_{j+i} \mid x_{1}, \ldots, x_{j-1}\right)\right)
\end{array}
$$

An extensive literature exists discussing many aspects of Copulas, Copula pair constructions and different types of vine copula. The interested reader is referred to [28] or the book of Mai and Scherer [29.

\section{A copula based approach for interval finite element computations}

\subsection{Interval finite element method}

The goal of an interval FE calculation is to find the bounds on the uncertainty in the model responses of eq. (1), given an interval description of the uncertainty in $\boldsymbol{x}^{\boldsymbol{I}} \in \mathcal{X}^{I} \subset \mathbb{R}^{d_{i}}$. For multiple parameters, the interval vector $\boldsymbol{x}^{\boldsymbol{I}}$ is defined as the Cartesian product of the intervals $x_{i}^{I}$ :

$$
\boldsymbol{x}^{\boldsymbol{I}}=x_{1}^{I} \times \ldots \times x_{d_{i}}^{I}
$$

and as such spans a hypercubic set by definition [1]. The interval FE method can as such be expressed as finding the solution set $\tilde{\mathbf{y}}$ :

$$
\tilde{\boldsymbol{y}}=\left\{\boldsymbol{y} \mid \boldsymbol{y}=\mathcal{M}(\boldsymbol{x}) ; \boldsymbol{x} \in \boldsymbol{x}^{I}\right\}
$$

Generally, $\tilde{\boldsymbol{y}}$ spans a non-convex manifold in $\mathbb{R}^{d_{o}}$, as the output responses $y_{i}$ are (possibly non-linearly) coupled through the PDE of the FE model under consideration. Therefore, instead of calculating the real uncertain solution 
set spanned by $\tilde{\mathbf{y}}$, the uncertainty at the output of the FE model generally is approximated using an interval vector $\boldsymbol{y}^{\boldsymbol{I}}$, which is usually calculated following a bounded optimisation problem, where the bounds $\underline{y}_{i}$ and $\bar{y}_{i}$ on each output quantity $y_{i}$ of the solution interval vector $\mathbf{y}^{I}$ are determined by searching the domain, defined by $\boldsymbol{x}^{\boldsymbol{I}}[1]$ :

$$
\begin{array}{ll}
\underline{y}_{i}=\min _{\boldsymbol{x} \in \boldsymbol{x}^{\boldsymbol{I}}} m_{i}(\boldsymbol{x}) & i=1, \ldots, d_{o} \\
\bar{y}_{i}=\max _{\boldsymbol{x} \in \boldsymbol{x}^{\boldsymbol{I}}} m_{i}(\boldsymbol{x}) & i=1, \ldots, d_{o}
\end{array}
$$

where $y_{i}^{I}=\left[\underline{y}_{i} ; \bar{y}_{i}\right]$ is the result interval scalar for the $i^{\text {th }}$ component of the solution interval vector of the model. This optimization problem has been shown to be solved with both local and global optimization algorithms [1. Solution of equation (17) returns the smallest hyper-cubic approximation $\boldsymbol{y}^{\boldsymbol{I}}$ of $\tilde{\boldsymbol{y}}$. Also methods that try to estimate $\tilde{\boldsymbol{y}}$ by its smallest convex set, [30, or based on affine arithmetic, have been proposed in recent years [31. Also convex hulls have been applied in this context [6, 7, 32.

\subsection{Bivariate dependence between intervals}

In a bivariate context, the concept of dependence can be illustrated using figure 1. This figure shows two arbitrary parameters $x_{1}$ and $x_{2}$. The corresponding uncertainty is scaled for both parameters to the interval $[0,1]$ for illustrative purposes, which is a straightforward operation on the data. In case no dependence between these parameters is taken into account, the space of admissible parameter combinations corresponds to the unit square $[0,1]^{2}$, which is also illustrated using the striped line. However, in case dependence is present between these two parameters, this can be modelled in an interval context by defining a set $\mathcal{D}_{12}$ that limits the range of admissible parameter combinations. This set is denoted the admissible set, and as such, the analysis becomes more generally set-theoretical (as intervals are a very specific type of convex sets). The degree of dependence can then be computed as the relative area of $\mathcal{D}_{12}$ with respect to $[0,1]^{2}[33$. 

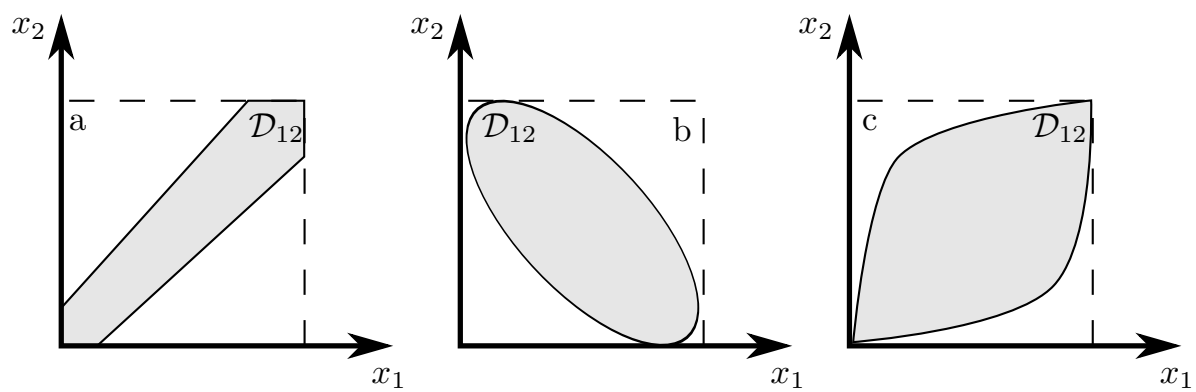

Figure 1: Illustration of dependence between two arbitrary parameters $x_{1}$ and $x_{2}$, modelled as a convex region $\mathcal{D}_{12}$.

The definition of $\mathcal{D}_{12}$ can be based on expert knowledge, first principle fundamentals, joint measurements of the uncertain parameters, etc. and $\mathcal{D}_{12}$ can take any shape within $[0,1]^{2}$, as long as it is physically relevant. For example, in an interval context, an admissible set that resembles the well-known diagonal band copula [34 can be applied as it requires only one additional parameter to be defined next to the interval uncertainty. In the specific case of figure 1 . in figure 1 a) a dependence is assumed that slightly decreases as $x_{1}$ and $x_{2}$ increase, figure 1 b) illustrates a negative ellipse-shaped dependence, as is for instance commonly applied in convex analysis [17. Finally figure 1 c) illustrates a large dependence in the centre of $[0,1]^{2}$ and small dependence around the extreme values of the interval. Evidently, this illustration of admissible sets is not exhaustive.

In the context of propagating this set-theoretical uncertainty, the optimization problem that is introduced in eq. (17) becomes a constrained optimization problem:

$$
\begin{array}{ll}
\underline{y}_{i}=\min _{\boldsymbol{x} \in \boldsymbol{x}^{\boldsymbol{I}}} m_{i}(\boldsymbol{x}) \quad i=1, \ldots, d_{o} \\
\text { s.t. } \quad \boldsymbol{x} \in \mathcal{D}_{12}
\end{array}
$$

In case $\mathcal{D}_{12}$ is a convex nodal set (i..e, a set that is defined by half-spaces), this reduces to a linearly inequality constrained optimization problem. In other cases, such as for instance an ellipsoidal admissible set, this becomes a non-linear constraint. If the underlying numerical model is sufficiently smooth, Newton- 
type optimizers can be applied to solve this problem as they are highly efficient [35. In other cases, when the model is for instance highly non-linear or bifurcated, semi-heuristic optimization algorithms such as Genetic optimizers or Particle Swarm algorithms have to be applied, which are in general less efficient. Application of both types of optimizers has been documented in the context of the propagation of interval algorithms [1, 36.

In case more than 3 parameters are considered, the definition of $\mathcal{D}$ might prove to be very cumbersome or even impossible, since this requires the definition of a $d_{i}>3$ dimensional convex set, which is intuitively speaking an impossible task. Therefore, a decomposition of the admissible set, based on the concept of copula pair construction is introduced in the next section.

\subsection{Admissible set decomposition}

In order to allow for a more flexible modelling of the joint-dependence structure of the interval uncertainty, captured by its admissible set, a similar pair construction as shown in eq. (7) and (12) is presented. Intuitively and loosely speaking, it can be argued that an admissible set $\mathcal{D}$ is some kind of piece-wise continuous copula, however without inferring any likelihood of certain parameter values within $\mathcal{D}$. The premise of this section is therefore that $\mathcal{D}$ can be similarly decomposed in the product of bivariate (conditional) $\mathcal{D}_{i j}$ and their marginal intervals.

However, the definitions of $\mathcal{D}$ and $\mathcal{C}$-vine copula, as presented respectively in eq. (13) and (14) cannot be translated directly to an interval context. A direct and naive translation would involve changing the random variables by intervals and the copula densities by bivariate admissible sets. This however is not advisable since intervals cannot track dependence throughout computations, and hence, this would inflate the interval bounds dramatically (see e.g., [1] for a discussion on this phenomenon). Instead, it is proposed to recast the decomposition into a product of inequality constraints bounding the search space of the optimization problem introduced in 17 from $\boldsymbol{x}^{\boldsymbol{I}}$ to $\mathcal{D}$.

Specifically, it is proposed to formulate the $\mathfrak{D}$-vine decomposition of the 
admissible set $\mathcal{D}_{1, \ldots, d_{i}}\left(x_{1}^{I}, x_{2}^{I}, \ldots, x_{d_{i}}^{I}\right)$ as:

$$
\mathcal{D}_{1, \ldots, d_{i}}\left(x_{1}^{I}, x_{2}^{I}, \ldots, x_{d_{i}}^{I}\right)=\bigotimes_{k=1}^{d_{i}} x_{k}^{I} \bigcap_{j=1}^{d_{i}-1} \bigcap_{i=1}^{d_{i}-j} \mathcal{D}_{i, i+j \mid i+1, \ldots, i+j+1}
$$

where the operator $\otimes$ is used to denote the Cartesian product of the intervals, and hence, the first part of the equation just describes the $d_{i}$ dimensional hyper-cube of independent intervals. The dependence is included by computing intersections of this hyper-cubic space with (conditional) bivariate admissible sets that are defined in analogy with the conditional bivariate copula densities in a probabilistic (vine-copula) approach. The $\mathfrak{D}$-vine decomposition is advantageous when the dependence structure of the admissible set is governed mostly by certain sets of piecewise-dependent parameter combinations. In that case, those can be modelled explicitly, whereas the higher order interactions between those parameters are separated.

Similarly, a $\mathfrak{C}$-vine decomposition is expressed as:

$$
\mathcal{D}_{1, \ldots, d_{i}}\left(x_{1}^{I}, x_{2}^{I}, \ldots, x_{d_{i}}^{I}\right)=\bigotimes_{k=1}^{d_{i}} x_{k}^{I} \bigcap_{j=1}^{d_{i}-1} \bigcap_{i=1}^{d_{i}-j} \mathcal{D}_{j, j+i \mid 1, \ldots, j-1}
$$

which is advantageous when the dependence of one parameter with all other parameters is rather easy to quantify [37. In that case, the lower-order dependence structure of the admissible set can be build completely around this central parameter.

As such, the admissible domain inside $\boldsymbol{x}^{I}$ can be fully described by a set of bivariate $\mathcal{D}_{i j}$. Since the definition of a two-dimensional admissible set is much more intuitive from an analysts point of view, this is easier as compared to defining the full $d_{i}$-dimensional $\mathcal{D}$. Furthermore, since the higher-order, conditional $\mathcal{D}_{i j \mid k}$ can be made a function of $x_{k}$, this allows for the highly flexible modelling of complex dependence structures in an interval context.

For a three-dimensional interval vector $\boldsymbol{x}^{I}=\left[x_{1}^{I}, x_{2}^{I}, x_{3}^{I}\right]$, the $\mathfrak{D}$-vine representation reduces to:

$$
\mathcal{D}\left(x_{1}^{I}, x_{2}^{I}, x_{3}^{I}\right)=x_{1}^{I} \times x_{2}^{I} \times x_{3}^{I} \cap \mathcal{D}_{12} \cap \mathcal{D}_{23} \cap \mathcal{D}_{13 \mid 2}
$$




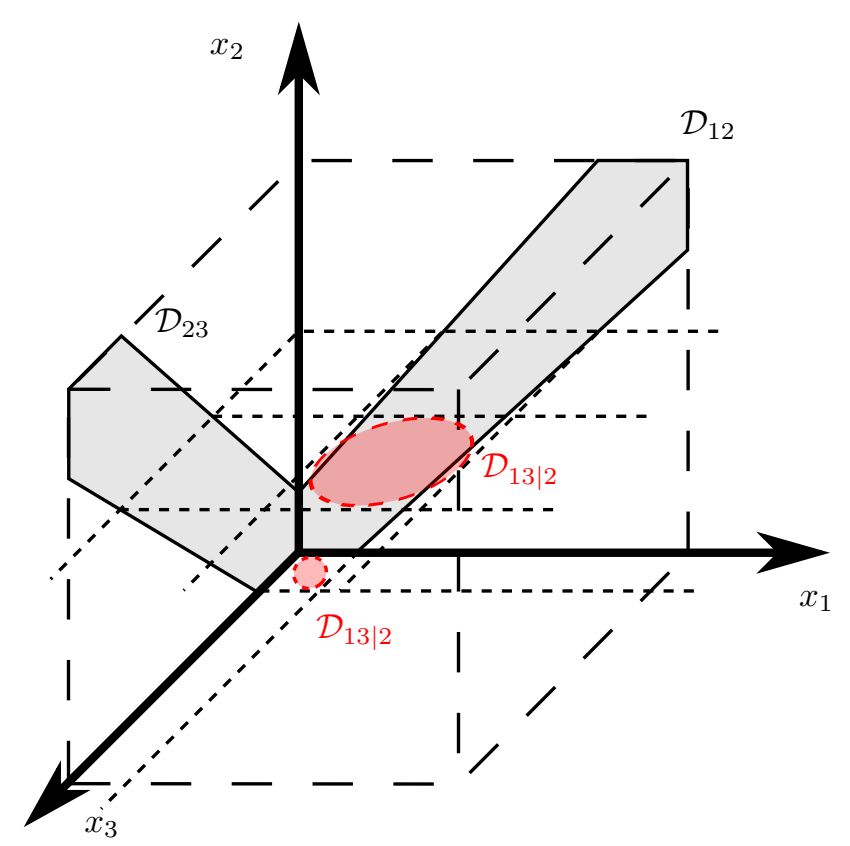

Figure 2: Decomposition of the $d=3$-dimensional dependence using the proposed admissible set decomposition via a $\mathfrak{D}$-vine structure.

205

with $\mathcal{D}_{i j}$ the bivariate dependence between $x_{i}$ and $x_{j}$. This concept is also illustrated in figure 2. This figure shows the unit cube $[0,1]^{3}$, together with $\mathcal{D}_{12}, \mathcal{D}_{23}$ and $\mathcal{D}_{13 \mid 2}$. As can be noted, the set $\mathcal{D}_{13 \mid 2}$ is not necessarily constant over $x_{2}$, allowing for the definition of highly complicated dependence structures with a very limited set of parameters.

Similarly, for a three-dimensional interval vector $\boldsymbol{x}^{I}=\left[x_{1}^{I}, x_{2}^{I}, x_{3}^{I}\right]$, the $\mathfrak{C}$-vine representation reduces to:

$$
\mathcal{D}\left(x_{1}^{I}, x_{2}^{I}, x_{3}^{I}\right)=x_{1}^{I} \times x_{2}^{I} \times x_{3}^{I} \cap \mathcal{D}_{12} \cap \mathcal{D}_{13} \cap \mathcal{D}_{23 \mid 1}
$$

with $\mathcal{D}_{i j}$ the bivariate dependence between $x_{i}$ and $x_{j}$. Note that for a threedimensional case, both $\mathfrak{D}$ - and $\mathfrak{C}$-vine structures give the same decomposition. Both representations allow to decouple any information that an analyst has on the dependence between combinations of interval-valued parameters from the actual magnitude of the uncertainty, and hence, are also in an interval context 
a very powerful tool.

Note that, while employing a similar decomposition structure as in a probabilistic context, the underlying meaning is fundamentally different. Instead of decomposing a multivariate density function, into its marginals and a set of bivariate copula, this method presents a way to limit the hypercube $x_{1}^{I} \times \ldots \times x_{d}^{I}$ of admissible values by a set of two-dimensional sets. While similar, still no inference of the likelihood of certain values within this set is made or aimed at. As such, the interval paradigm is not violated following this approach. Furthermore, the presented method can be regarded as a generalization of the work on dependence between non-probabilistic quantities presented in e.g., [5] or [14]. For instance, when all bi-variate admissible sets are considered ellipsoidal, one would end up with a high-dimensional ellipsoid.

The presented method is especially of practical interest in case an analyst has quantified information about bivariate dependence between two quantities in the model. For instance, consider the case of additively manufactured components. In those parts, underlying physical process parameters jointly affect mechanical quantities such as strength and stiffness, but also dimensional quantities such as the part thickness (see e.g., [38]). Instead of trying to quantify and model the full dependence structure of all these uncertain quantities at once, the analyst can focus on quantifying a limited set of bivariate dependence structures, and constructing the full-dimensional dependence from these. As a second example of application, when an analyst is currently faced with say, 20 uncertain model quantities, ranging from bolt connection stiffness values to localized masses and support stiffness values (as is the case in e.g., the DLR AIRMOD structure [2]), (s)he either can opt to consider all parameters independent and work hyper-cubic via the classical interval paradigm or assume a similar dependence structure among all parameters, and employ classical convex set approaches. Following this approach, the analyst can make e.g., directed measurements of certain parameters of interest and fit a certain convex structure to those (in analogy to the work presented in [18, 19]), use expert knowledge to model other dependencies as ellipses, and so on. Hence, a more flexible approach 
for the modelling of dependence is provided by considering this decomposition of $\mathcal{D}$ in bivariate admissible sets.

\section{Propagation of the admissible set}

This section presents two approaches to propagate the admissible set through a numerical model. The first approach converts the well-known global optimization approach into a constraint global optimization approach that is able to account for the dependence between the model responses. The second approach extends the Transformation Method, as presented by Hanss in [30] towards the propagation of (set-theoretical) dependent intervals.

\subsection{Global optimization}

In the context of propagating this uncertainty through the numerical model, this decomposition serves as a constraint for the global optimization that underlies the interval finite element model. Specifically, to infer the bounds on $y$, following set of constrained optimization problems is solved:

$$
\begin{array}{ll}
\underline{y}_{i}=\min _{\boldsymbol{x} \in \boldsymbol{x}^{\boldsymbol{I}} m_{i}(\boldsymbol{x})} & \text { s.t. } \boldsymbol{x} \in \mathcal{D}\left(x^{I}\right) \\
\bar{y}_{i}=\max _{\boldsymbol{x} \in \boldsymbol{x}^{I} m_{i}(\boldsymbol{x})} & \text { s.t. } \boldsymbol{x} \in \mathcal{D}\left(x^{I}\right)
\end{array}
$$

for $i=1, \ldots, d_{o}$, which can be solved by most Newton-type or semi-heuristic optimization algorithms. Moreover, since the convex hulls are only 2-dimensional, the corresponding computational overhead for evaluating these equality constraints is very limited [6].

\subsection{The enriched transformation method}

Alternatively, when monotonic models $\mathcal{M}$ are considered, als a semi-analytic approach can be applied. Instead of handling the problem as a black-box, the intersections of the bivariate admissible sets with the hyper-cubic interval space are computed and the nodes of the resulting polytopes are propagated through the FE model. The necessary steps to perform these computations are described in the following section. 
As a first step, the hyper-cubic input uncertainty $\boldsymbol{x}^{I}=x_{1}^{I} \times x_{2}^{I} \times x_{d_{i}}^{I}$ is represented as a set of linear inequalities:

$$
\boldsymbol{x}^{\boldsymbol{I}} \equiv A_{h c} x-b_{h c} \geq 0
$$

with $A_{h c} \in \mathbb{R}^{h_{x} \times d_{i}}, x \in \mathbb{R}^{d_{i}}$ and $b_{h c} \in \mathbb{R}^{h_{x}}$. Herein, $h_{x}$ is the number of linear inequalities that are necessary to bound the admissible set. According to Minkowski-Weyl's theorem both representations are equivalent.

Then, similarly to eq. (25), each bivariate admissible set from eq. 19) or eq. 20 is represented as a set of 2-dimensional linear inequalities:

$$
\mathcal{D}_{\mathcal{I}(i)} \equiv A_{\mathcal{I}(i)} x-b_{\mathcal{I}(i)} \geq 0 \quad i=1, \ldots, d_{i}\left(d_{i}-1\right) / 2
$$

with $A \in \mathbb{R}^{h_{x} \times 2}, x \in \mathbb{R}^{2}$ and $b_{i} \in \mathbb{R}^{h_{x}}$. $\mathcal{I}(i)$ is an index set containing the indices of the $d_{i}\left(d_{i}-1\right) / 2$ conditional and unconditional bivariate admissible sets. Each of these sets describes the dependence in a two-dimensional intersection of the full admissible set $\mathcal{D}$. As such, $\mathcal{D}$ can be obtained by asserting that admissible parameter values should satisfy the linear inequalities in each of these intersections.

In a first step, only the unconditional bivariate admissible sets are considered. Since not each $\mathcal{D}_{\mathcal{I}(i)}$ contains information on the same $x_{i}, i=1, \ldots, d_{i}$, these projections have to be assembled as follows:

$$
\mathcal{D}_{u} \equiv A_{u} \boldsymbol{x}-b_{u} \geq 0
$$


with $A_{u}=$

$\left[\begin{array}{ccccc}a_{h c}\left(1, x_{1}\right) & a_{h c}\left(1, x_{2}\right) & \ldots & a_{h c}\left(1, x_{d_{i}-1}\right) & a_{h c}\left(1, x_{d_{i}}\right) \\ a_{h c}\left(2, x_{1}\right) & a_{h c}\left(2, x_{2}\right) & \ldots & a_{h c}\left(2, x_{d_{i}-1}\right) & a_{h c}\left(2, x_{d_{i}}\right) \\ \vdots & \vdots & \vdots & \vdots & \vdots \\ a_{h c}\left(h_{s}, x_{1}\right) & a_{h c}\left(h_{s}, x_{2}\right) & \ldots & a_{h c}\left(h_{s}, x_{d_{i}-1}\right) & a_{h c}\left(h_{s}, x_{d_{i}}\right) \\ a_{\mathcal{I}(1)}\left(1, x_{1}\right) & a_{\mathcal{I}(1)}\left(1, x_{2}\right) & \ldots & 0 & 0 \\ a_{\mathcal{I}(1)}\left(2, x_{1}\right) & a_{\mathcal{I}(1)}\left(2, x_{2}\right) & \ldots & 0 & 0 \\ \vdots & \vdots & \vdots & \vdots & \vdots \\ a_{\mathcal{I}(1)}\left(h_{12}, x_{1}\right) & a_{\mathcal{I}(1)}\left(h_{12}, x_{2}\right) & \ldots & 0 & 0 \\ \vdots & \vdots & \vdots & \vdots & \vdots \\ 0 & 0 & \cdots & a_{\mathcal{I}\left(d_{i}-1\right)}\left(h_{\mathcal{I}\left(d_{i}-1\right)}, x_{d_{i}-1}\right) & a_{\mathcal{I}\left(d_{i}-1\right)}\left(h_{\mathcal{I}\left(d_{i}-1\right)}, x_{d_{i}}\right)\end{array}\right]$

and $b_{u}$ :

$$
b_{f u l l}=\left[\begin{array}{c}
b_{h c}(1) \\
b_{h c}(2) \\
\vdots \\
b_{h c}\left(d_{i}\right) \\
b_{\mathcal{I}(1)}(1) \\
b_{\mathcal{I}(1)}(2) \\
\vdots \\
b_{\mathcal{I}(1)}\left(d_{i}\right) \\
\vdots \\
b_{\mathcal{I}\left(d_{i}-1\right)}\left(d_{i}\right)
\end{array}\right]
$$

In case only unconditional admissible sets are defined, the space of all admissible $\boldsymbol{x} \in \mathcal{D}$ is fully determined. This is for instance the case when only pair-wise dependence structures need to be constructed. The final step is then to propagate all vertices of $\mathcal{D}$ through $\mathcal{M}$. The realization set $\tilde{\boldsymbol{y}}$ is then obtained as:

$$
\tilde{\boldsymbol{y}}=\{\boldsymbol{y} \mid \boldsymbol{y}=\mathcal{M}(\boldsymbol{x}) ; \boldsymbol{x} \in \mathcal{D}\}
$$

where the bounds of the set are constructed using linear interpolation between 
the propagated nodes.

When also conditional admissible sets are included in the analysis, further intersections of $\mathcal{D}_{u}$ have to be computed sequentially. For instance, to compute the conditional admissible set $\mathcal{D}_{13 \mid 2}$, the parameter space is first uniformly discretized over $x_{2}$ into $n$ bins:

$$
x_{2}=\left\{x_{2}^{1}, x_{2}^{2}, \ldots, x_{2}^{n}\right\}
$$

with:

$$
x_{2}^{2}-x_{2}^{1}=\frac{\bar{x}_{2}-\underline{x}_{2}}{n}
$$

providing slices of $\mathcal{D}_{u}$ for each $x_{2}^{e}$. Then, each of this slices is intersected with the the corresponding conditional admissible set. Those slices are finally recombined to reconstruct the admissible set $\mathcal{D}$ including the conditional admissible sets.

It should be noted that the computational cost of the method can scale badly for large scale problems and complicated dependence structures. The application of the transformation method for independent intervals already requires $2^{d_{i}}$ deterministic function evaluations. In case dependence is included in the analysis, only more vertices of the admissible set need to be propagated through $\mathcal{M}$ and hence, those calculations can become expensive. A priori estimation of the increase in computational cost is highly non-trivial. Indeed, the computational cost is directly related to the number of vertices in $\mathcal{D}$, which in its turn is dependent on the number of bivariate admissible sets that are included in the analysis, as well as the level of their mutual dependence.

Application of surrogate modeling techniques such as Kriging [39, 40, or Artificial Neural Networks 2, 41 have, among many other techniques (see e.g., [1] for a recent overview), already proven their merit in the context of interval computations. 


\section{Case study 1: analytical function}

As a first example, dependent interval uncertainty is propagated through a simple analytical equation having three parameters $x_{i}, i=1, \ldots, 3$ :

$$
y=x_{1} \cdot x_{2}-2 \cdot x_{3}
$$

with $x_{1}^{I}=[1,4], x_{2}^{I}=[2,6], x_{3}^{I}=[3,5]$.

This function is constructed such that the extreme values for $y$ do not correspond with either $\underline{x}$ or $\bar{x}$. The dependence between these parameters assumes following structure:

$$
\begin{aligned}
\mathcal{D}_{12} & =\mathcal{H}\left(\left|x_{2}+x_{1}-1\right|-\theta_{1}\right) \\
\mathcal{D}_{23} & =\mathcal{H}\left(\left|x_{1}-x_{2}\right|-\theta_{2}\right) \\
\mathcal{D}_{13 \mid 2} & =\mathcal{H}\left(\left|x_{3}\left(x_{2}\right)-x_{1}\left(x_{2}\right)\right|-\theta_{3}\left(x_{2}\right)\right)
\end{aligned}
$$

with $\mathcal{H}$ the Heaviside function, $\theta_{i}$ a measure for the dependence and || denoting the absolute value. For the construction of the admissible set $\mathcal{D}$ the problem is first scaled to the unit cube $[0,1]^{3}$. The tested values for the dependency are listed in table 1 . The $\hat{\bullet}$ operator indicates the interval that yields the extreme values for $y$.

The admissible set $\mathcal{D}$, as illustrated in eq. (19), is constructed based on eqns. (34) - (36). The enriched transformation method is applied to discretise $\mathcal{D}$ into a set of vertices, which then are used to propagate the dependent intervals. To construct the admissible sets that are conditional on $x_{2}$, the domain $x_{2}$ is discretised in 100 elements. The computation of the intersections and conversion of the convex hulls into half-spaces, the Matlab FEX package Analyse $N$-dimensional Polyhedra in terms of Vertices or (In)Equalities was used.

As can be noted, the results without dependence between the interval parameters are highly over-conservative with respect to the case when the dependence is taken into account following the proposed set-theoretical approach. The degree of conservatism decreases when the parameters in $\boldsymbol{\theta}$ are increased, as this increases the dependence between the intervals by reducing the size of $\mathcal{D}$. Furthermore, it can be noted that the necessary number of function evaluations 
increases with the number of bivariate admissible sets that are taken into account. Especially inclusion of conditional admissible sets leads to a significant increase in number of function evaluations, which is due to the discretisation of the conditional axis. Post-processing of the assembled admissible set, e.g., by performing regression on the bounding half-spaces, could solve this issue. This is however outside the scope of this paper.

Table 1: Results of the propagation of the interval uncertainty and admissible set in eq. 33

\begin{tabular}{lllll}
\hline $\boldsymbol{\theta}=\left[\theta_{1}, \theta_{2}, \theta_{3}\right]$ & $\underline{\hat{\boldsymbol{x}}}$ & $\hat{\overline{\boldsymbol{x}}}$ & $y^{I}$ & \# eval. \\
\hline$[0,0,0]$ & {$[1,2,5]$} & {$[4,6,3]$} & {$[-8,18]$} & 8 \\
{$[0.25,0,0]$} & {$[1,3,5]$} & {$[4,5,3]$} & {$[-6,14]$} & 12 \\
{$[0,0.25,0]$} & {$[1,3,5]$} & {$[4,6,3.5]$} & {$[-7,17]$} & 12 \\
{$[0.25,0.25,0]$} & {$[1,3,5]$} & {$[4,5,3]$} & {$[-7,14]$} & 14 \\
{$[0.25,0.25,0.25]$} & {$[1,3,4.5]$} & {$[4,5,3.5]$} & {$[-6,13]$} & 700 \\
{$[0.5,0,0]$} & {$[1,4,5]$} & {$[4,4,3]$} & {$[-6,10]$} & 12 \\
{$[0,0.5,0]$} & {$[1,4,5]$} & {$[4,6,4]$} & {$[-6,16]$} & 12 \\
{$[0.5,0.5,0]$} & {$[1,4,5]$} & {$[4,4,3]$} & {$[-6,10]$} & 12 \\
{$[0,0,0.5]$} & {$[1,2,4]$} & {$[4,6,4]$} & {$[-6,16]$} & 700 \\
{$[0.5,0.5,0.5]$} & {$[1,4,4]$} & {$[3.75,4.33,4.08]$} & {$[-4,8.08]$} & 700 \\
{$[0.75,0,0]$} & {$[1,5,5]$} & {$[4,3,3]$} & {$[-5,6]$} & 12 \\
{$[0,0.75,0]$} & {$[1,2,3.5]$} & {$[464.5]$} & {$[-5,15]$} & 700 \\
{$[0.75,0.75,0]$} & {$[1,5,5]$} & {$[4,3,3]$} & {$[-5,6]$} & 14 \\
{$[0.75,0.75,0.75]$} & {$[1,5,4.25]$} & {$[3.625,3.5,4]$} & {$[-3.5,4.687]$} & 700 \\
\hline
\end{tabular}

As was explained in section 3 the higher order dependence terms can be made a function of the conditional parameter. Table 2 illustrates some computations that were made using a hypothetical dependence of $\mathcal{D}_{13 \mid 2}$. As can be noted, a highly flexible modelling of the admissible set is possible.

This dependence structure corresponding to the last case listed in table 2 is illustrated in figure 3 . Since the parameter describing the dependence in $\mathcal{D}_{13 \mid 2}$ varies according to a trigonometric description, this set is not convex. This 
Table 2: Results of the propagation of the interval uncertainty and admissible set in eq. 33. with $\theta_{3}^{1}\left(x_{2}\right)=\left(1-\exp \frac{x_{2}}{\max \left(x_{2}\right)}\right) /\left(1-\exp \frac{\overline{x_{2}}}{\max \left(x_{2}\right)}\right.$ and $\theta_{3}^{2}(\omega)=\sin (4 * \omega-p i / 2), \omega=0, \ldots, 4 * p i$

\begin{tabular}{llll}
\hline $\boldsymbol{\theta}=\left[\theta_{1}, \theta_{2}, \theta_{3}\right]$ & $\underline{\hat{\boldsymbol{x}}}$ & $\hat{\overline{\boldsymbol{x}}}$ & $y^{I}$ \\
\hline$\left[0,0, x_{2}\right]$ & {$[1,2,5]$} & {$[4,6,5]$} & {$[-8,14]$} \\
{$\left[0.5,0.5, x_{2}\right]$} & {$[1,4,4]$} & {$[4,4,4]$} & {$[-4,8]$} \\
{$\left[0,0, \theta_{3}^{1}\left(x_{2}\right)\right]$} & {$[1,2,5]$} & {$[4,6,4.9]$} & {$[-8,14.2]$} \\
{$\left[0.5,0.5, \theta_{3}^{1}\left(x_{2}\right)\right]$} & {$[1,4,4.279]$} & {$[4,4,3.721]$} & {$[-4.558,8.558]$} \\
{$\left[0.75,0.5, \theta_{3}^{2}(\omega)\right]$} & {$[1,5.5,5]$} & {$[3.62,3.5,3]$} & {$[-4.5,6.68]$} \\
\hline
\end{tabular}

visualization is obtained by propagating a Sobol Sequence containing $1 \cdot 10^{06}$ samples through the analytical function, discarding the values that do not comply with the admissible set, and computing the alpha-shape representation of the resulting data. This figure illustrates that also non-convex $\mathcal{D}$ are obtainable following the proposed approach. Note that such explicit computation is only needed for visualization purposes, as the necessary computations of the indicator functions are made for each step of an iteration of the optimization solvers. Since this case study is only three-dimensional, no explicit difference between $\mathfrak{D}$ and $\mathfrak{C}$-vine pair constructions is included in the study, as both are in this case analogous.

\section{Case study 2: composite blade}

\subsection{Case introduction}

The second case study concerns a finite element model of a long and slender blade. The structure has a total length of $30 \mathrm{~m}$ and the width is $1 \mathrm{~m}$ at the widest part. This blade is produced using a multilayer laminar composite material, with deterministic ply material properties $E_{1}=231 G P a, E_{2}=77 G P a, \nu_{12}=0.31$ and $G_{12}=G_{23}=G_{13}=42.7 \mathrm{GPa}$. Different lay-ups are placed in the structure, where close to the attachment of the blade (left-most), the lay-up is thicker as compared to at the end-point (right-most). The blade consists of a composite outside shell (top, leading edge, bottom, trailing edge), as well as two vertical 


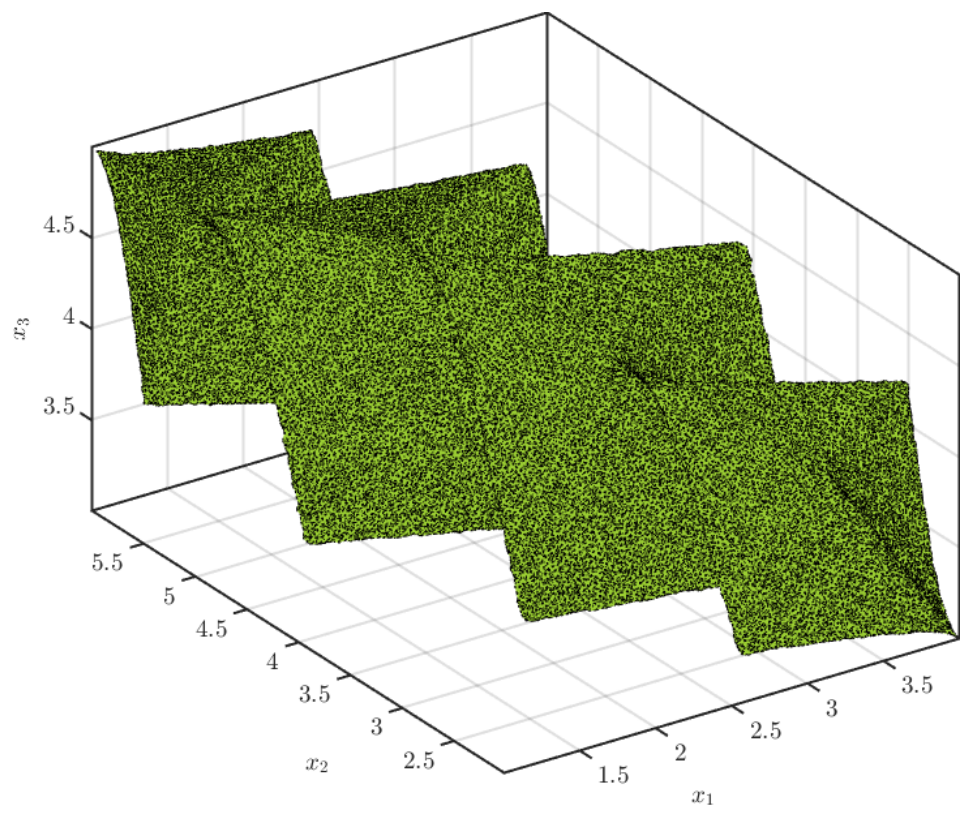

Figure 3: Illustration of the dependence structure of $\theta_{3}^{2}(\omega)$ used to model the dependence between 3 intervals. Comment: A Matlab live figure will be provided with the final version of the paper.

stiffening ribs in the centre. A total of 15 different composite lay-ups are present in the blade, which are summarized in table 3 .

The dynamic behaviour of the structure is modelled using a Finite Element model containing 621 nodes, 606 bilinear shell elements, 573 rigid connections, 10 concentrated masses and 132 rod elements. The left end of the composite blade is fixed rigidly. The finite element model of this structure is shown in figure 4

The model is solved for its 10 first eigenmodes and corresponding resonance frequencies. Table 4 lists the result of the deterministic simulation. The effect of mode-crossover and -veering is accounted for by tracking the mode shapes via the modal assurance criterion.

The uncertainty the analyst has concerning the true values of the primary and secondary Young's modulus $\left(E_{1}\right.$ and $\left.E_{2}\right)$, as well as the ply thickness in the red, yellow and blue areas indicated in figure $4\left(t_{1}, t_{2}\right.$ and $\left.t_{3}\right)$, is modelled 
Table 3: Composite lay-up structure of the blade. Left means at $y=0$ in figure 4 and the leading edge is depicted at the back side of figure 4

\begin{tabular}{|c|c|c|}
\hline Location & Lay-up (symmetrical) & $\begin{array}{l}\text { thickness } \\
\text { per layer }(\mathrm{mm})\end{array}$ \\
\hline Top and bottom left & $+-45^{0}$ & $1.5 \mathrm{~mm}$ \\
\hline leading edge left & $+-45^{0}$ & $1.5 \mathrm{~mm}$ \\
\hline front-middle edge left & $+-+-+-+45^{0}$ & $1.5 \mathrm{~mm}$ \\
\hline back-middle vertical left & $+-+-+-+45^{0}$ & $1.5 \mathrm{~mm}$ \\
\hline trailing edge left & $+-+-+-+45^{0}$ & $1.5 \mathrm{~mm}$ \\
\hline Top and bottom middle & $+-45^{0}$ & $1.5 \mathrm{~mm}$ \\
\hline leading edge middle & $+-45^{0}$ & $1.5 \mathrm{~mm}$ \\
\hline front-middle edge middle & $+-+-+-+45^{0}$ & $1.5 \mathrm{~mm}$ \\
\hline back-middle vertical middle & $+-+-+-+45^{0}$ & $1.5 \mathrm{~mm}$ \\
\hline trailing edge middle & $+-+-+-+45^{0}$ & $1.5 \mathrm{~mm}$ \\
\hline Top and bottom right & $+-45^{0}$ & $1.5 \mathrm{~mm}$ \\
\hline leading edge right & $+-45^{0}$ & $1.5 \mathrm{~mm}$ \\
\hline front-middle edge right & $+-+-+-+45^{0}$ & $1.5 \mathrm{~mm}$ \\
\hline back-middle vertical right & $+-+-+-+45^{0}$ & $1.5 \mathrm{~mm}$ \\
\hline trailing edge right & $+-+-+-+45^{0}$ & $1.5 \mathrm{~mm}$ \\
\hline
\end{tabular}

Figure 4: Finite element model of the composite blade 
Table 4: Deterministic eigenmodes and -frequencies of the composite blade

\begin{tabular}{lll}
\hline Mode number & description & $\mathrm{f}$ \\
\hline 1 & $1^{\text {st }}$ vertical bending & $0.79 \mathrm{~Hz}$ \\
2 & $1^{\text {st }}$ horizontal bending & $2.07 \mathrm{~Hz}$ \\
3 & $2^{\text {nd }}$ vertical bending & $3.11 \mathrm{~Hz}$ \\
4 & $3^{\text {th }}$ vertical bending & $7.72 \mathrm{~Hz}$ \\
5 & $2^{\text {nd }}$ horizontal bending & $8.67 \mathrm{~Hz}$ \\
6 & $4^{\text {th }}$ vertical bending & $14.45 \mathrm{~Hz}$ \\
7 & $3^{\text {th }}$ horizontal bending & $21.68 \mathrm{~Hz}$ \\
8 & $5^{\text {th }}$ vertical bending & $23.84 \mathrm{~Hz}$ \\
9 & $1^{\text {st }}$ torsion & $26.76 \mathrm{~Hz}$ \\
10 & $6^{\text {th }}$ vertical bending & $35.01 \mathrm{~Hz}$ \\
\hline
\end{tabular}

as intervals. Specifically, these intervals are defined as $E_{1}=[190,200] G P a$, $E_{2}=[70,77] G P a, t_{1}=[0.012,0.015] \mathrm{mm}, t_{2}=[0.015,0.017] \mathrm{mm}$ and $t_{3}=$ $[0.011,0.018]$.

\subsection{Artificial Neural Network meta-modelling}

A single forward computation of the model takes about 20 seconds of wallclock time on a high laptop equipped with $32 \mathrm{~Gb}$ or RAM and an Intel Core i7-7700HQ CPU @ $2.8 \mathrm{GHz}$. In case independent intervals are propagated, 32 deterministic model evaluations are needed. However, as is clear from table 1 this number can increase quickly when dependence is included in the analysis. Furthermore, since a comparison of the Extended Transformation Method with a Genetic Algorithm is performed, computational expenses can become high when such a global optimization is performed with the full FE model. Therefore, to limit the computational expense, a 4-layer sigmoid-symmetric Artificial Neural Network (ANN) with (5:10:1)-configuration is trained for each eigenfrequency and compiled to $\mathrm{C}++$ for computational efficiency. The lay-out of the network is iteratively chosen where a maximum performance with a minimum 
of hidden nodes is aimed at. Hereto, a training dataset containing 1750 samples is generated using Latin Hypercube Sampling between the interval bounds of the model uncertainty. Furthermore, a validation data set of 750 specimens was used to verify the accuracy of the trained ANN. To prevent over-training, Bayesian regulation back-propagation was used [42, which expresses the ANN model performance $P$ as:

$$
P=\frac{\xi}{d} \sum_{i=1}^{d}\left(y_{\text {training }, i}-y_{A N N, i}\right)^{2}+\frac{\chi}{d} \sum_{i=1}^{d} w_{i}^{2}
$$

with $\xi$ and $\chi$ the regularisation parameters and $y_{\text {training, } i}$ and $y_{A N N, i}$ respectively the responses that are captured in the training data set, and the predicted responses of the ANN. When $\xi>>\chi$, the network will drive the mean squared error to a lower value. Conversely, when $\chi>>\xi$, the network weights and biasses will be smaller as compared to a non-regularised performance function, forcing the network response to be smoother. Hence, the former case tends towards a perfect representation of the training data, albeit with the risk of performing bad on new data, whereas the latter aims at a better generalisation performance of the ANN. Specifically, this training is performed following a Bayesian approach, where the weights $w$ and biasses $b$ are modelled as random variables, and identified following a Bayesian approach that minimises $P$. The regularisation parameters $\xi$ and $\chi$ are related to the variances of the random weights and biases, and are also found by performing Bayesian estimation 43, 44. These computations are performed using the Neural Network toolbox in Matlab. The performance of the ANN on both the training and validation data set is shown 5 for each eigenfrequency. As may be noted, a highly performing set of meta-models is obtained, and hence, they can be used to make viable predictions about the model behaviour at strongly reduced cost.

\subsection{D-vine decomposition}

A first illustration of the admissible set decomposition follows the $\mathfrak{D}$-vine approach. This corresponds to the case where the analyst has direct knowledge about dependence between $E_{1}^{I}, E_{2}^{I}$ on the one hand and $t_{1}^{I}, t_{2}^{I}$ and $t_{3}^{I}$ on the other 


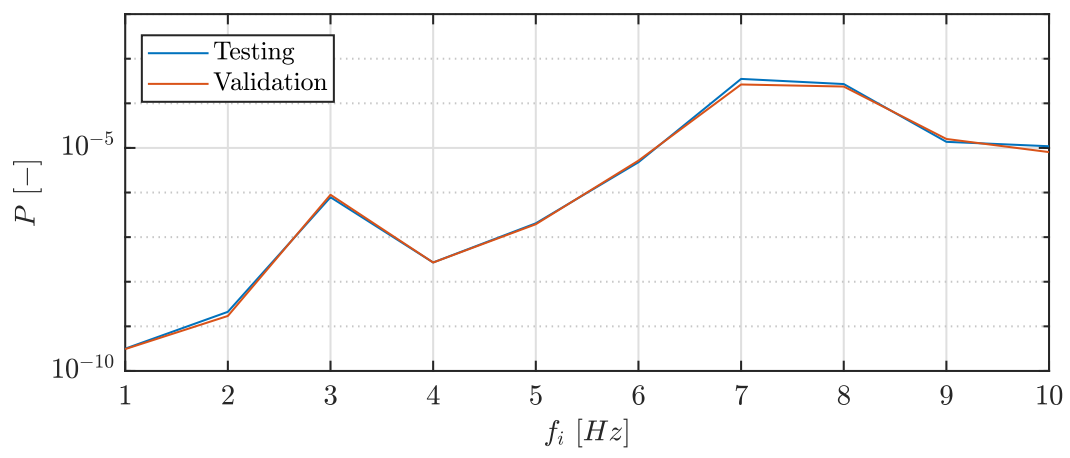

Figure 5: Performance of the ANN on the training and validation data set

hand. Specifically, the $\mathfrak{D}$-vine decomposition of the admissible set of parameters $\mathcal{D}\left(E_{1}^{I}, E_{2}^{I}, t_{1}^{I}, t_{2}^{I}, t_{3}^{I}\right)$ can be written explicitly as:

$$
\begin{aligned}
\mathcal{D}\left(E_{1}^{I}, E_{2}^{I}, t_{1}^{I}, t_{2}^{I}, t_{3}^{I}\right)= & E_{1}^{I} \times E_{2}^{I} \times t_{1}^{I} \times t_{2}^{I} \times t_{3}^{I} \cap \\
& \mathcal{D}_{12} \cap \mathcal{D}_{23} \cap \mathcal{D}_{34} \cap \mathcal{D}_{45} \cap \\
& \mathcal{D}_{13 \mid 2} \cap \mathcal{D}_{24 \mid 3} \cap \mathcal{D}_{35 \mid 4} \cap \\
& \mathcal{D}_{14 \mid 23} \cap \mathcal{D}_{25 \mid 34} \cap \\
& \mathcal{D}_{15 \mid 234}
\end{aligned}
$$

${ }_{388}$ When only pairwise dependence between $E_{1}^{I}, E_{2}^{I}$ on the one hand and $t_{1}^{I}, t_{2}^{I}$ 
is considered, the different terms in eq. (38) are given in this case study by:

$$
\begin{aligned}
\mathcal{D}_{12} & =\mathcal{H}\left(\left|E_{2}+E_{1}-1\right|-\theta_{1}\right) \\
\mathcal{D}_{23} & =E_{2}^{I} \times t_{1}^{I} \\
\mathcal{D}_{34} & =\mathcal{H}\left(\left|t_{1}-t_{2}\right|-\theta_{2}\right) \\
\mathcal{D}_{45} & =\mathcal{H}\left(\left|t_{2}-t_{3}\right|-\theta_{3}\right) \\
\mathcal{D}_{13 \mid 2} & =E_{1 \mid E_{2}}^{I} \times t_{1 \mid E_{2}}^{I} \\
\mathcal{D}_{24 \mid 3} & =E_{2 \mid t_{1}}^{I} \times t_{2 \mid t_{1}}^{I} \\
\mathcal{D}_{35 \mid 4} & =t_{t_{1} \mid t_{2}}^{I} \times t_{t_{3} \mid t_{2}}^{I} \\
\mathcal{D}_{14 \mid 23} & =E_{1 \mid E_{2} t_{1}}^{I} \times t_{2 \mid E_{2} t_{1}}^{I} \\
\mathcal{D}_{25 \mid 34} & =E_{2 \mid t_{1} t_{2}}^{I} \times t_{3 \mid t_{1} t_{2}}^{I} \\
\mathcal{D}_{15 \mid 234} & =E_{1 \mid E_{2} t_{1} t_{2}}^{I} \times t_{3 \mid E_{2} t_{1} t_{2}}^{I}
\end{aligned}
$$

with $\theta_{i}$ a measure for the dependence between the interval parameters 9 . In this case study, $\boldsymbol{\theta}=[0.5 ; 0.9 ; 0.7]$. These values are chosen purely for illustrative purposes. The corresponding admissible sets correspond to the case where $E_{1}$ and $E_{2}$ have a negative dependence and $t_{1}, t_{2}$ and $t_{3}$ have a positive dependence. Physically, the former could be explained by unmodelled uncertainty on the fibre-matrix mixture ratio in the composite material, yielding a negative dependence between these two Young's moduli. The positive dependence between the thickness values on the other hand can for instance originate from some systematic but unknown offset in the lay-up process. As can be noted, the higher order terms are not included, as they are all represented by a Cartesian product. Note than any kind of dependence structure can be applied for the bivariate admissible sets. The result of propagating $\mathcal{D}$ is compared to propagating a 5-dimensional hyper-cubic input set.

The cross-sections of this 5 -dimensional convex dependence region are shown in figure 6. The blue area is the domain covered by the independent intervals, whereas the orange area corresponds to the admissible set that is defined. AS can be noted, the method allows for the independent modelling of the depen- 


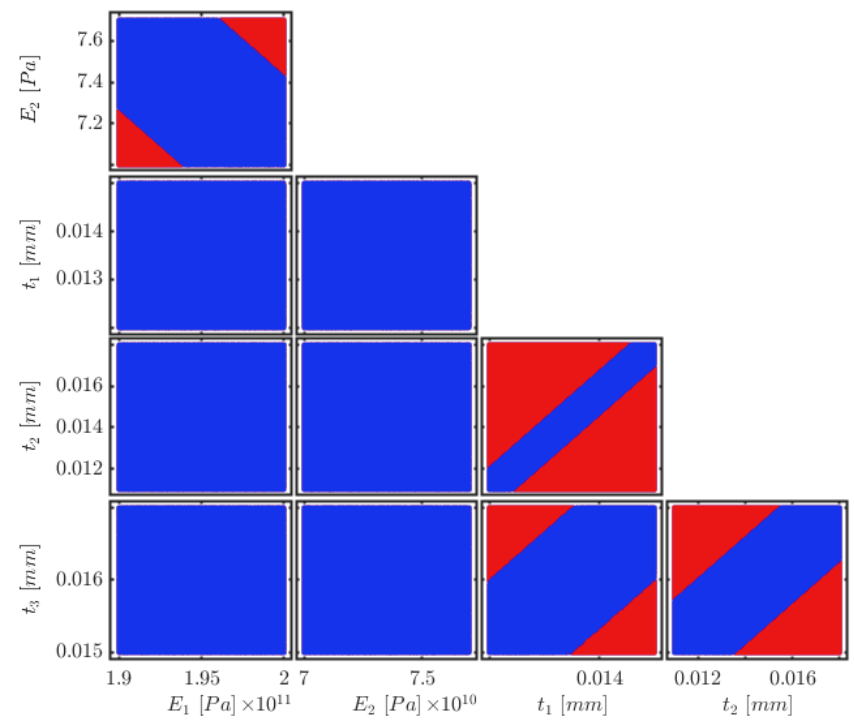

Figure 6: 2-dimensional intersections of the 5-dimensional admissible set $\mathcal{D}$. Red: hyper-cubic space covered by the independent intervals. Blue: intersections of the admissible set.

dence between the Young's moduli and the thickness values by selecting the appropriate decomposition structure and corresponding values.

space covered by the independent intervals. Blue: intersections of the admissible set.

Based on this decomposed set, the optimisation problem that is introduced in eq. 24 is solved for the 10 first resonance frequencies of the composite blade.

This is specifically obtained by means of a Genetic Algorithm that starts from an initial uniform distribution between the interval bounds consisting of 50 samples. An elite count of 3 was used, together with a forward migration factor of 0.2 , a Gaussian mutation function and a cross-over fraction of 0.8 . The algorithm is deemed to be converged when the improvement of the objective function over 50 subsequent generations is smaller than $1 \cdot 10^{6}$. Such optimization is performed for each bound of each resonance frequency. Hence, 20 optimization procedures should be performed. On average, one call to the Genetic Algorithm solver requires $\mathcal{O}\left(10^{4}\right)$ deterministic function evaluations for this specific FE model.

20 Making use of the ANN meta-models and parallel processing, this is well within 
feasible computational cost.

The result of this optimization procedure is illustrated in figure 7 . This figure shows two-dimensional cross-sections of the 10-dimensional result manifold, obtained by propagating 50000 Sobol samples from the dependent input parameters. As can be noted, when more dependence is included in the analysis, the solution manifold becomes smaller and smaller. Furthermore, also the dependence between the resonance frequencies is impacted.

The result of the optimization runs is illustrated in this figure as green crosses. As is clear, the bounded optimization problem yields the exact (hypercubic) bounds on the eigenfrequency.

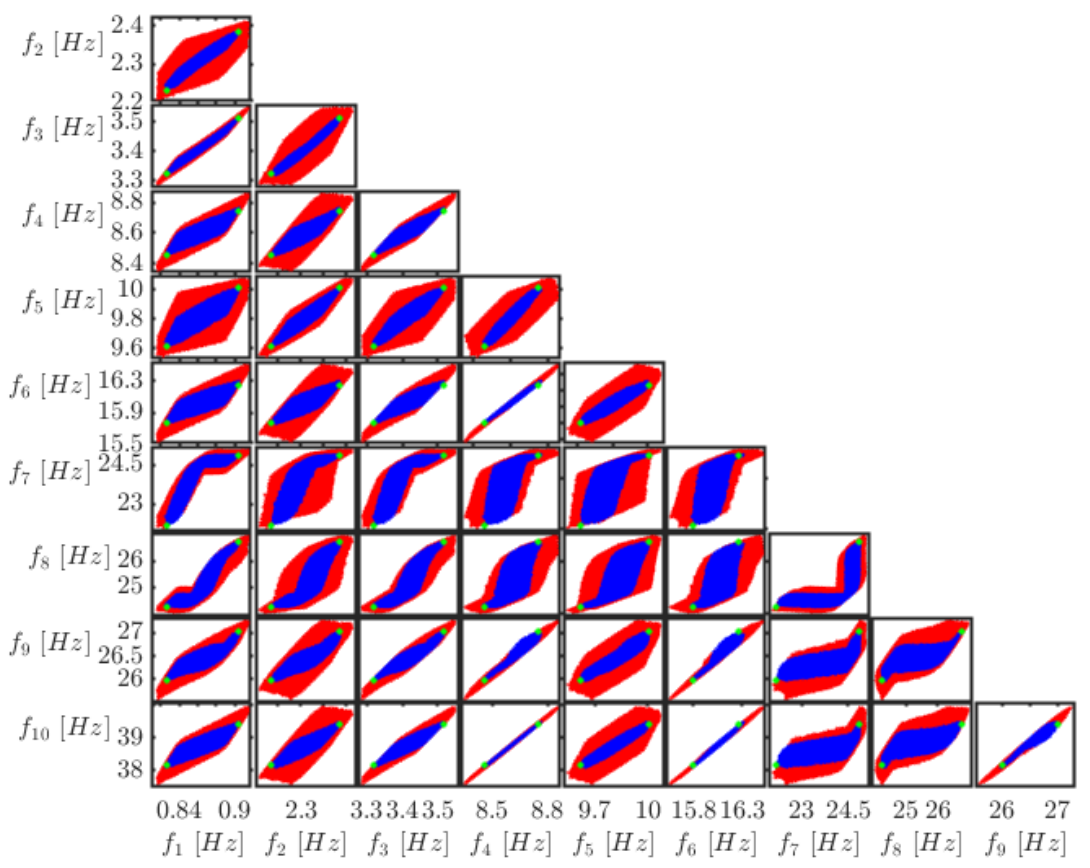

Figure 7: 2-dimensional intersections of the 10-dimensional eigenfrequency space. Red: result of propagating the independent intervals. Blue: result of propagating the dependent intervals via the admissible set $\mathcal{D}$ with the Extended Transformation Method. Green dots: result of propagating the dependent intervals via the admissible set $\mathcal{D}$ via Global Optimization. 
The decomposition of $\mathcal{D}$ according to a $\mathfrak{C}$-vine decomposition is straightforward and can be performed in full analogy to the presented case studies.

\section{Conclusions}

This paper presents a flexible approach for the modelling of dependent intervals for multivariate input spaces. Specifically, it is proposed to construct the dependence structure in a similar approach to copula pair constructions, yielding a limited set of 2-dimensional dependence functions. Also, the wellknown transformation method is extended to account for dependence between multiple intervals. A first case study, where the developed method is applied to an analytical function is included to illustrate the main ideas. Application of the enriched transformation method indicates that by introducing dependence between the model parameters, the width of the output interval is decreased significantly. The second case study applies the methodology to a realistic finite element model of a long, slender composite blade. Two different dependence structures are propagated and it is shown that the method is well capable of limiting the set of admissible parameter combinations, yielding tighter output sets. However, the computational cost of propagating the dependent intervals, both via global optimization as the enriched transformation method scales badly with the dimension of the input space, but also with the nature of the dependence. Application of surrogate modelling was used to alleviate this problem.

\section{Acknowledgements}

The authors would like to acknowledge the financial support of the Flemish research foundation in the framework of the research project G0C2218N and the post-doctoral grant $12 \mathrm{P} 359 \mathrm{~N}$ of Matthias Faes. The authors also would like to thank the reviewers for the constructive criticism and remarks. 


\section{Bibliography}

[1] D. Moens, M. Hanss, Non-probabilistic finite element analysis for parametric uncertainty treatment in applied mechanics: Recent advances, Finite Elements in Analysis and Design 47 (1) (2011) 4-16. doi:10.1016/j. finel.2010.07.010

[2] M. Faes, M. Broggi, E. Patelli, Y. Govers, J. Mottershead, M. Beer, D. Moens, A multivariate interval approach for inverse uncertainty quantification with limited experimental data, Mechanical Systems and Signal Processing 118 (2019) 534-548. doi:10.1016/j.ymssp.2018.08.050. URL https://doi.org/10.1016/j.ymssp.2018.08.050https://www. sciencedirect.com/science/article/pii/S0888327018305946? via\{\%\}3Dihub

[3] R. L. Muhanna, R. L. Mullen, Uncertainty in mechanics problems - interval-based approach, Journal of Engineering Mechanics 127 (6) (2001) 557-566.

[4] A. Sofi, E. Romeo, A novel Interval Finite Element Method based on the improved interval analysis, Computer Methods in Applied Mechanics and Engineering 311 (2016) 671-697. doi:http://dx.doi.org/10.1016/j. cma.2016.09.009.

[5] W. Verhaeghe, W. Desmet, D. Vandepitte, D. Moens, Interval fields to represent uncertainty on the output side of a static FE analysis, Computer Methods in Applied Mechanics and Engineering 260 (0) (2013) 50-62. doi : $10.1016 / \mathrm{j} . \mathrm{cma} .2013 .03 .021$

[6] M. Faes, D. Moens, Identification and quantification of spatial interval uncertainty in numerical models, Computers and Structures 192 (2017) 16-33. doi:10.1016/j.compstruc.2017.07.006.

[7] M. Faes, J. Cerneels, D. Vandepitte, D. Moens, Identification and quantification of multivariate interval uncertainty in finite element models, Com- 
puter Methods in Applied Mechanics and Engineering 315 (2017) 896-920. doi:10.1016/j.cma.2016.11.023

[8] M. Broggi, M. Faes, E. Patelli, Y. Govers, D. Moens, M. Beer, Comparison of bayesian and interval uncertainty quantification: Application to the airmod test structure, in: 2017 IEEE Symposium Series on Computational Intelligence (SSCI), 2017, pp. 1-8. doi:10.1109/SSCI.2017.8280882.

[9] M. Imholz, D. Vandepitte, D. Moens, Derivation of an Input Interval Field Decomposition Based on Expert Knowledge Using Locally Defined Basis Functions, in: 1st ECCOMAS Thematic Conference on International Conference on Uncertainty Quantification in Computational Sciences and Engineering, 2015, pp. 1-19.

[10] M. Imholz, D. Vandepitte, D. Moens, Analysis of the effect of uncertain clamping stiffness on the dynamical behaviour of structures using interval

field methods 807 (2015) 195-204. doi:10.4028/www.scientific.net/ AMM.807.195.

[11] M. Faes, D. Moens, Identification and quantification of spatial variability in the elastostatic properties of additively manufactured components, in: 19th AIAA Non-Deterministic Approaches Conference, no. January, 2017, pp. 1-13. doi:10.2514/6.2017-1771.

URL http://arc .aiaa.org/doi/10.2514/6.2017-1771

[12] A. Sofi, G. Muscolino, I. Elishakoff, Static response bounds of Timoshenko beams with spatially varying interval uncertainties, Acta Mechanica 226 (11) (2015) 3737-3748. doi:10.1007/s00707-015-1400-9.

[13] D. Wu, W. Gao, Hybrid uncertain static analysis with random and interval fields, Computer Methods in Applied Mechanics and Engineering 315 (2017) 222-246. doi:10.1016/j.cma.2016.10.047.

[14] L. P. Zhu, I. Elishakoff, J. H. Starnes, Derivation of multi-dimensional ellipsoidal convex model for experimental data, Mathematical and Com- 
$512 \quad$ puter Modelling 24 (2) (1996) 103-114. doi:http://dx.doi.org/10.

$513 \quad 1016 / 0895-7177(96) 00094-5$

${ }_{514}$ [15] X. Wang, I. Elishakoff, Z. Qiu, Experimental data have to decide which of the nonprobabilistic uncertainty descriptions - convex modeling or interval analysis - to utilize, Journal of Applied Mechanics 75 (4) (2008) 41018. doi:10.1115/1.2912988.

[16] X. Wang, I. Elishakoff, Z. Qiu, C. Kou, Hybrid theoretical, experimental and numerical study of vibration and buckling of composite shells with scatter in elastic moduli, International Journal of Solids and Structures 46 (13) (2009) 2539-2546.

[17] I. Elishakoff, Y. Bekel, Application of Lamé's Super Ellipsoids to Model Initial Imperfections, Journal of Applied Mechanics 80 (6) (2013) 61006. doi:10.1115/1.4023679.

[18] I. Elishakoff, N. Sarlin, Uncertainty quantification based on pillars of experiment, theory, and computation. Part I: Data analysis, Mechanical Systems and Signal Processing 74 (2015) 54-72. doi:10.1016/j.ymssp.2015.04. 035 .

[19] I. Elishakoff, N. Sarlin, Uncertainty quantification based on pillars of experiment, theory, and computation. Part I: Data analysis, Mechanical Systems and Signal Processing 74 (2015) 54-72. doi:10.1016/j.ymssp.2015.04. 035.

[20] M. Sklar, Fonctions de repartition an dimensions et leurs marges, Publ. Inst. Statist. Univ. Paris 8 (1959) 229-231.

[21] J. Behrensdorf, M. Broggi, M. Beer, Reliability analysis of networks interconnected with copulas, in: Proceedings of the 3rd International Symposium on Uncertainty Quantification and Stochastic Modeling, Florianopolis, Brazil, 2018. 
[22] R. Jane, L. D. Valle, D. Simmonds, A. Raby, A copula-based approach for the estimation of wave height records through spatial correlation, Coastal Engineering 117 (2016) 1 - 18. doi:https: //doi.org/10.1016/j.coastaleng.2016.06.008 URL http://www.sciencedirect.com/science/article/pii/ S0378383916301193

[23] M. Tian, D.-Q. Li, Z.-J. Cao, K.-K. Phoon, Y. Wang, Bayesian identification of random field model using indirect test data, Engineering Geology 210 (2016) 197-211.

[24] A. J. Patton, Copula-based models for financial time series, in: Handbook of financial time series, Springer, 2009, pp. 767-785.

[25] G. Elidan, Copulas in machine learning, in: Copulae in mathematical and quantitative finance, Springer, 2013, pp. 39-60.

[26] T. Bedford, R. M. Cooke, Probability density decomposition for conditionally dependent random variables modeled by vines, Annals of Mathematics and Artificial intelligence 32 (1-4) (2001) 245-268.

[27] T. Bedford, R. M. Cooke, Vines: A new graphical model for dependent random variables, The Annals of Statistics 30 (4) (2002) 1031-1068.

URL http://www . jstor.org/stable/1558694

[28] K. Aas, C. Czado, A. Frigessi, H. Bakken, Pair-copula constructions of multiple dependence, Insurance: Mathematics and economics 44 (2) (2009) 182-198.

[29] M. Scherer, Mai, Simulating copulas: stochastic models, sampling algorithms, and applications, Vol. 6, \# N/A, 2017.

[30] M. Hanss, The transformation method for the simulation and analysis of systems with uncertain parameters, Fuzzy Sets and Systems 130 (3) (2002) 277-289. doi:10.1016/S0165-0114(02)00045-3. 
[31] G. Muscolino, A. Sofi, Stochastic analysis of structures with uncertain-butbounded parameters via improved interval analysis, Probabilistic Engineer-

ing Mechanics 28 (2012) 152-163. doi:10.1016/j .probengmech.2011.08. 011.

[32] M. Faes, J. Cerneels, D. Vandepitte, D. Moens, Identification of interval fields for spatial uncertainty representation in finite element models, in: V. P. M. Papadrakakis, V. Papadopoulos, G. Stefanou (Ed.), Proceedings of the ECCOMAS Congress 2016, Crete, Greece, 2016.

[33] O. Giannini, M. Hanss, An interdependency index for the outputs of uncertain systems, Fuzzy Sets and Systems 159 (11) (2008) 1292-1308. doi:10.1016/j.fss.2007.12.028

[34] W. Verhaeghe, W. Desmet, D. Vandepitte, I. Elishakoff, D. Moens, Bounding the dependence measures for spatial uncertainties, in: M. Vorechovský, V. Sadílek, S. Seitl, V. Veselý, R. L. Muhanna, R. L. Mullen (Eds.), 5th International Conference on Reliable Engineering Computing (REC 2012), 2012, pp. 599-612.

[35] J. Nocedal, S. J. Wright, Numerical Optimization (1999) 625doi:10.1007/ b98874.

[36] I. Boulkaibet, T. Marwala, M. I. Friswell, H. H. Khodaparast, S. Adhikari, Fuzzy finite element model updating using metaheuristic optimization algorithms, arXiv preprint arXiv:1701.00833.

[37] C. Czado, U. Schepsmeier, A. Min, Maximum likelihood estimation of mixed c-vines with application to exchange rates, Statistical Modelling 12 (3) (2012) 229-255.

[38] M. Pavan, M. Faes, D. Strobbe, B. V. Hooreweder, T. Craeghs, D. Moens, W. Dewulf, On the influence of inter-layer time and energy density on selected critical-to-quality properties of $\{$ PA12 $\}$ parts produced via laser 
sintering, Polymer Testing 61 (2017) 386 - 395. doi:https://doi.org/ 10.1016/j.polymertesting.2017.05.027.

[39] J. D. Martin, T. W. Simpson, Use of Kriging Models to Approximate Deterministic Computer Models, AIAA Journal 43 (4) (2005) 853-863. doi:10.2514/1.8650

[40] M. De Munck, D. Moens, W. Desmet, D. Vandepitte, An efficient response surface based optimisation method for non-deterministic harmonic and transient dynamic analysis, CMES - Computer Modeling in Engineering and Sciences 47 (2) (2009) 119-166. doi:10.3970/cmes.2009.047.119

[41] Z. Deng, Z. Guo, X. Zhang, Interval model updating using perturbation method and radial basis function neural networks, Mechanical Systems and Signal Processing 84 (2017) 699-716.

[42] H. B. Demuth, M. H. Beale, O. De Jess, M. T. Hagan, Neural Network Design, 2nd Edition, Martin Hagan, USA, 2014.

[43] D. J. MacKay, Bayesian interpolation, Neural Comput. 4 (3) (1992) 415447.

[44] F. D. Foresee, M. T. Hagan, Gauss-newton approximation to bayesian learning, in: Neural Networks, 1997., International Conference on, Vol. 3, IEEE, 1997, pp. 1930-1935. 